\title{
Group Homotopy Algorithm with a Parameterized Newton Iteration for Symmetric Eigen Problems
}

\author{
Ran Baik ${ }^{1}$, Karabi Datta ${ }^{2}$ and Yoopyo Hong ${ }^{2}$ \\ 1 Department of Computer Engineering, Honam University, \\ Gwangju 506-090, Korea \\ 2 Department of Mathematical Sciences, Northern Illinois University, \\ DeKalb, IL 60115, USA \\ baik@honam.ac.kr \\ \{dattak, hong\}@math.niu.edu
}

\begin{abstract}
In this paper we develop a general Homotopy method called the Group Homotopy method to solve the symmetric eigenproblem. The Group Homotopy method overcomes notable drawbacks of the existing Homotopy method, namely, (i) the possibility of breakdown or having a slow rate of convergence in the presence of clustering of the eigenvalues and (ii) the absence of any definite criterion to choose a step size that guarantees the convergence of the method. On the other hand, the Group Homotopy method maintains attractive features of the ordinary Homotopy method such as the natural parallelism and the structure preserving properties.
\end{abstract}

\section{Introduction}

Let $S$ and $A$ be two real $n \times n$ symmetric matrices. Define a mapping $H$ : $\mathbb{R} \longrightarrow M_{n}$ such that $H(t)=A(t) \equiv(1-t) S+t A, \quad t \in[0,1]$. Note that $A(t)=S+t(A-S)$ so that the matrix $A(t)$ can be made close to $S$ by choosing $t$ small enough. The objective is to obtain the set of all the eigenpairs $\left\{\left(\begin{array}{c}u_{k} \\ \lambda_{k}\end{array}\right)\right\}, k=1, \ldots, n$ of $A(1)=A$ by successively obtaining the set of eigenpairs $\left\{\left(\begin{array}{c}u_{k}(i) \\ \lambda_{k}(i)\end{array}\right)\right\}, k=1, \ldots, n$ of $A\left(t_{i}\right), 0=t_{0}<t_{1}<\cdots<t_{n}=1$, starting from the set of eigenpairs $\left\{\left(\begin{array}{c}x_{k} \\ \alpha_{k}\end{array}\right)\right\}, k=1, \ldots, n$ of $A(0)=S[1,2,3,6,7,8]$. The procedure is called the Homotopy method. A major problem that arises in implementing a Homotopy method for the eigenproblem is: how to choose $\Delta t_{i} \equiv t_{i+1}-t_{i}$ properly so that the all eigenpairs of $A\left(t_{i}+\Delta t_{i}\right)$ can be obtained from the eigenpairs (possibly clustered) of $A\left(t_{i}\right)$ using Newton's method with a reasonable number of iterations. In this paper we provide an answer to the question. It is well known that there exists a small neighborhood of each eigenpair of $A\left(t_{i+1}\right)$ such that Newton's method converges in the small neighborhood. 
Thus the question is: how close should $A\left(t_{i}\right)$ be to $A\left(t_{i+1}\right)$ to have a guarantee that each eigenpair of $A\left(t_{i}\right)$ is in that convergence neighborhood of the corresponding eigenpair of $A\left(t_{i+1}\right)$ under Newton's method. [4] provides an answer to above question whenever the eigenvalues of $A\left(t_{i}\right)$ are not clustered. We define $\operatorname{gap}^{*}\left(A\left(t_{i}\right)\right) \equiv \min _{k}\left\{\operatorname{gap}_{k}\left(A\left(t_{i}\right)\right)\right\}$ where $\operatorname{gap}_{k}\left(A\left(t_{i}\right)\right)=\left|\lambda_{k}(i)-\lambda_{k+1}(i)\right|$, and $\lambda_{k}(i)$ are the eigenvalues of $A\left(t_{i}\right)$ in decreasing order for $k=1, \ldots, n-1$. We show that if $\Delta t_{i}$ is chosen such that $\Delta t_{i} \leq \frac{g a p^{*}\left(A\left(t_{i}\right)\right)}{q}$ for some $q \geq 4$, then all eigenpairs of $A\left(t_{i}\right)$ will converge to the corresponding eigenpairs of $A\left(t_{i}+\Delta t_{i}\right)$ under Newton's method. The method will fail when $\operatorname{gap}^{*}\left(A\left(t_{i}\right)\right)$ becomes extremely small for some $t_{i} \in[0,1)$, that is, the matrix $A\left(t_{i}\right)$ has the clustered eigenvalues. To overcome the problem we introduce the group gap, Gap* in section 2. It is noted that under the group gap we always obtain a sufficiently large step size $\Delta t_{i}$ so that the Homotopy method terminates in a finite number of steps. We also discuss problems that may occur due to the large step size $\Delta t_{i}$ when the Newton's method is applied to obtain the successive eigenpairs. We show in section 3 that this problem can be easily resolved by a simple modification of the Newton's method. The new Homotopy method with group gap, Gap* is called the Group Homotopy method. The Group Homotopy method with the parameterized Newton's iteration never fails and is guaranteed to give all eigenpairs of $A$ in a finite number of steps. Furthermore, the choice of the initial matrix $S$ can be arbitrary even though a good choice of the initial matrix will facilitate the convergence. An algorithm of the Group Homotopy method is presented in section 3 .

\section{Properties of the Group Homotopy Method}

In this section we develop a method called the Group Homotopy method which is a generalization of the Individual Homotopy Method. This is done to overcome the difficulties that arise in the Individual Homotopy method. Assume that an arbitrary real symmetric matrix $S \in M_{n}$ is chosen as the initial matrix. As we have observed previously, the Individual Homotopy method fails whenever the gap between some adjacent eigenvalues of the matrix $A\left(t_{i}\right)$ becomes quite small. This situation may occur when some eigenvalues of $A\left(t_{i}\right)$ are clustered, i.e., there are groups of a consecutive eigenvalues in which the distance between any two groups is much larger than the gaps among the eigenvalues that belong to the same group. We resolve the difficulty by using a concept, called the group gap. For that purpose, we define the clustering of eigenvalues as follows:

Definition 1. Let $\sigma(A)=\left\{\lambda_{i}\right\}_{i=1, \ldots, n}$ be the set of eigenvalues of a symmetric matrix $A$ in decreasing order. We say that two adjacent eigenvalues $\lambda_{k}$ and $\lambda_{k+1}$ are clustered if $\left|\lambda_{k}-\lambda_{k+1}\right|<$ Gap $^{*}, 1 \leq k \leq n-1$ where Gap* is a given positive number. 
Definition 2. We say that a subset $G=\left\{\lambda_{k}, \lambda_{k+1}, \ldots, \lambda_{k+m}\right\} \subseteq \sigma(A)$ is a group of clustering of eigenvalues if $\left|\lambda_{k+i}-\lambda_{k+i+1}\right|<G a p^{*}$ for all $i=0, \ldots, m-$ 1 where $\left|\lambda_{k-1}-\lambda_{k}\right| \geq$ Gap $^{*}$ and $\left|\lambda_{k+m}-\lambda_{k+m+1}\right| \geq$ Gap $^{*}$.

Now we define the Group Homotopy Method. Let $S$ and $A$ be given real $n \times n$ symmetric matrices such that $S$ is the initial matrix and $A$ is the target matrix. Then $A\left(t_{i+1}\right)=A\left(t_{i}\right)+\Delta t_{i}(A-S)$ where $0 \leq t_{0}<t_{1}<\ldots<t_{n}=1$, $\Delta t_{i}=t_{i+1}-t_{i}, A\left(t_{0}\right)=S$ and $A\left(t_{n}\right)=A$.

Definition 3. We say a Homotopy method is the Group Homotopy method if the step size $\Delta t_{i}$ is determined by Gap*.

Before we develop the Group Homotopy method we make the following brief remarks.

\section{Remarks}

(A)Advantages of using Gap*: The stepsize $\Delta t_{i}=\frac{g a p^{*}\left(A\left(t_{i}\right)\right)}{q}$ in the Individual Homotopy are determined completely by the eigenvalues of $A\left(t_{i}\right)$ where the eigenpath of the Homotopy is determined a'priori by the initial matrix $S$. On the other hand Gap* in the Group Homotopy is a constant that we are free to choose to group the eigenvalues of $A\left(t_{i}\right)$ so that the resulting stepsize $\Delta t_{i}$ is large enough.

(B) How to choose Gap*: For the choice of $G_{a p}^{*}$, one can consider the following: (1) the dimension of matrices involved, (2) magnitude of the eigenvalues of matrices involved, and (3) the nearness of the initial matrix to the target matrix. Furthermore, a good choice of Gap* should be the one that can be computed easily while providing a large enough step size for the Homotopy method to terminate in a reasonable amount of time. The following choice of Gap $^{*}$ is used in this paper: $G_{a p}^{*}=\delta\|A-S\|=\delta\|A-S\|, \delta>0$ where $\|\cdot\|$ is a matrix norm.

(C) Justification of the choice $G a p^{*}=\delta\|A-S\|$ : We will justify our choice of $\mathrm{Gap}^{*}$ and show how it leads to a simple determination of $\Delta t_{i}$. For some positive scalars $c_{1}$ and $c_{2}$, we have $\|A-S\|^{2}=c_{1}\|A-S\|_{F}^{2}=c_{1} c_{2} \sum_{k=1}^{n}\left(\alpha_{k}-\lambda_{k}\right)^{2}$ where $\alpha_{k}$ 's and $\lambda_{k}$ 's are the eigenvalues of the matrices $S$ and $A$, respectively. Thus, $\delta\|A-S\|$ measures overall nearness of the initial eigenvalues to the target eigenvalues. It also implicitly considers the eigenvectors of both matrices $A$ and $S$. Set $\Delta t_{i}=\frac{G a p^{*}}{q\|A-S\|}, q>0$. Note that $\Delta t_{i}=\frac{G a p^{*}}{q\|A-S\|}=\frac{\delta\|A-S\|}{q\|A-S\|}=\frac{\delta}{q}$ for some $\delta>0$. Thus if we set $\delta=\operatorname{gap}^{*}\left(A\left(t_{i}\right)\right)$ then $\Delta t_{i}=\frac{\operatorname{gap}^{*}\left(A\left(t_{i}\right)\right)}{q}$, or the Group Homotopy method simply reduces to the previous Individual Homotopy method. Furthermore, note that if $\delta$ is chosen to be a positive number then $\Delta t_{i}=\frac{\delta}{q}$ is a fixed constant, regardless of $i$. Thus under the Group Homotopy the total number of steps is not more than $\left[\frac{q}{\delta}\right]$, a nearest integer larger than 
$\frac{q}{\delta}$. When we have a good choice of initial matrix $S$, we might set $\Delta t=1$. It is precisely the case when $q\|A-S\|<\frac{g a p^{*}(S)}{q}$, for some $q>0$. In this case if we set Gap $^{*}=q\|A-S\|$ then $\Delta t=1$ and $\operatorname{Gap}^{*}<\frac{g a p^{*}(S)}{q}, q>0$ implies each group of clustering eigenvalues of $S=A(0)$ consists of exactly one eigenvalue of $S$. It is also easy to construct an example of the case where a group of the clustering eigenvalues contains all eigenvalues of $A\left(t_{i}\right)$ in the Group Homotopy.

\subsection{Properties of the Gap*}

Suppose symmetric matrices $A$ and $S$ are given, Gap $^{*}=\delta\|A-S\|, \delta>0$. Then $\Delta t=\frac{\delta}{q}, q>0$. Since $\left\|A\left(t_{i+1}\right)-A\left(t_{i}\right)\right\|_{2} \leq\left\|A\left(t_{i+1}\right)-A\left(t_{i}\right)\right\|$ for any matrix norm $\|\cdot\|$ where $\|\cdot\|_{2}$ is the spectral matrix norm, $\left\|A\left(t_{i+1}\right)-A\left(t_{i}\right)\right\|_{2}=\Delta t\|A-S\|_{2}=$ $\frac{\delta}{q}\|A-S\|_{2} \leq \frac{\delta}{q}\|A-S\|=\frac{G a p^{*}}{q}, q \geq 0$. Since the inequality holds for any matrix norm $\|\cdot\|$, we use the Frobenius matrix norm, $\|\cdot\|_{F}$ to compute Gap ${ }^{*}$ in the examples provided in section 3 , i.e.,

$$
\left\|A\left(t_{i+1}\right)-A\left(t_{i}\right)\right\|_{2}<\frac{G a p^{*}}{q}
$$

where Gap $^{*}=\delta\|A-S\|_{F}$, for some $\delta>0$.

Suppose the eigenvalues of $A\left(t_{i}\right)$ are partitioned as groups of clustering eigenvalues; $\sigma\left(A\left(t_{i}\right)\right)=\cup_{k=1}^{g} G_{k}$, where $G_{k}=\left\{\lambda_{k_{1}}(i) \geq \cdots \geq \lambda_{k_{s_{k}}}(i)\right\}$ is a group of clustering eigenvalues of $A\left(t_{i}\right), s_{1}+\cdots+s_{g}=n$. We partition the eigenvalues of $A\left(t_{i+1}\right)$ conformally to form the groups correspond to the grouping in $\sigma\left(A\left(t_{i}\right)\right)$, $\sigma\left(A\left(t_{i+1}\right)\right)=\cup_{k=1}^{g} G_{k}^{\prime}$, where $G_{k}^{\prime}=\left\{\lambda_{k_{1}}(i+1) \geq \cdots \geq \lambda_{k_{s_{k}}}(i+1)\right\}, s_{1}+\cdots+$ $s_{g}=n$. We call $G_{k}^{\prime}$ the counterpart of $G_{k}$.

Lemma 4. Suppose $B, C \in M_{n}$ are symmetric matrices and let $\lambda_{1} \geq \cdots \geq \lambda_{n}$ and $\alpha_{1} \geq \cdots \geq \alpha_{n}$ be the eigenvalues of $B$ and $C$, respectively. Then $\|B-C\|_{2}=$ $\rho(B-C) \geq \max _{k}\left\{\left|\lambda_{k}-\alpha_{k}\right|\right\}$, where $\rho(B-C)=\max _{k}\left\{\left|\lambda_{k}(B-C)\right| / \lambda_{k}(B-\right.$ $C)$ are the eigenvalues of $(B-C)\}$. Consequently, if $\|B-C\|_{2} \leq \frac{\text { Gap }^{*}}{q}$, for $q>0$, then $\left|\lambda_{k}-\alpha_{k}\right| \leq \frac{\text { Gap }^{*}}{q}$ for all $k=1, \cdots, n$.

From Lemma 4 we have $\max _{k}\left\{\left|\lambda_{k}(i+1)-\lambda_{k}(i)\right|\right\} \leq \frac{\text { Gap }^{*}}{q}$ where $\lambda_{k}(i+1)$ and $\lambda_{k}(i)$ are the eigenvalues of $A\left(t_{i+1}\right)$ and $A\left(t_{i}\right)$, respectively. Therefore, the $k$ th eigenvalue of $A\left(t_{i+1}\right)$ must be within $\frac{G a p^{*}}{q}$ distance from the $k$ th eigenvalue of $A\left(t_{i}\right)$ for all $k=1, \ldots, n$.

Theorem 5. Let $G \subseteq \sigma\left(A\left(t_{i}\right)\right)$ be a group of clustering eigenvalues and $G^{\prime}$ be the counterpart in $\sigma\left(A\left(t_{i+1}\right)\right)$. Then 
(i) $\left|\lambda_{j}(i)-\lambda_{j}(i+1)\right| \leq \frac{G a p^{*}}{q}$ for $\lambda_{j}(i) \in G$ and $\lambda_{j}(i+1) \in G^{\prime}$,

(ii) $\left|\lambda_{s}(i)-\lambda_{t}(i+1)\right| \geq\left(1-\frac{1}{q}\right) G a p^{*}$ for $\lambda_{s}(i) \in G$ and $\lambda_{t}(i+1) \notin G^{\prime}$,

(iii) $\left|\lambda_{s}(i+1)-\lambda_{t}(i+1)\right| \geq\left(1-\frac{2}{q}\right)$ Gap $^{*}$ for $\lambda_{s}(i+1) \in G^{\prime}$ and $\lambda_{t}(i+1) \notin G^{\prime}$

Implications of Theorem 5: Suppose that two successive matrices $A\left(t_{i}\right)$ and $A\left(t_{i+1}\right)$ are obtained via group gap $G_{a p} *$ in the process of the Group Homotopy. Then the statement (i) asserts that the $j$ th eigenvalue in a group $G^{\prime} \subseteq \sigma\left(A\left(t_{i+1}\right)\right)$ must be within $\frac{G a p^{*}}{q}$ distance from the $j$ th eigenvalue in $G \subseteq \sigma\left(A\left(t_{i}\right)\right)$. That is for a large enough $q>0$, the eigenvalues can change only a small amount between two successive steps of the Group Homotopy method.

Statement (ii) shows that there is a big enough gap between the eigenvalues in $G$ and the eigenvalues that are not in the counterpart $G^{\prime}$. Thus we see that the eigenvalues in $G$ are more likely to converge to the eigenvalues in $G^{\prime}$ under the Newton iteration. From the statement (iii) we see that for a large enough $q>0$ there is a gap between any two $G^{\prime}$ 's in $\sigma\left(A\left(t_{i+1}\right)\right)$ that are counterparts of groups in $\sigma\left(A\left(t_{i}\right)\right)$. Thus a group of the clustered eigenvalues tends to remain as a group of the clustered eigenvalues during the Group Homotopy.

\section{Group Homotopy Algorithm}

Now we prove the main result of the Group Homotopy with the parameterized Newton's iteration. In the following we let $\sigma\left(A\left(t_{i}\right)\right)=\left\{\alpha_{1}, \ldots, \alpha_{n}\right\}, \alpha_{n} \leq \cdots \leq$ $\alpha_{1}$ and $\sigma\left(A\left(t_{i+1}\right)\right)=\left\{\lambda_{1}, \ldots, \lambda_{n}\right\}, \lambda_{n} \leq \cdots \leq \lambda_{1}$ for the notational convenience. A Parametrized Newton Iteration :[5] Suppose $\left(\begin{array}{c}x \\ \alpha\end{array}\right) \in \mathbb{R}^{n+1}$ is given and let $A \in M_{n}$ be a symmetric matrix. Assume $\alpha \notin \sigma(A)$.

Then for $l=0,1, \ldots$, define $x^{(l+1)}=\frac{1}{\hat{\beta}^{(l)}}\left(\alpha^{(l)} I-A\right)^{-1} x^{(l)}, \alpha^{(l+1)}=\alpha^{(l)}-\frac{\beta^{(l)}}{\left(\hat{\beta}^{(l)}\right)^{2}}$ where $\beta^{(l)}=x^{(l) T}\left(\alpha^{(l)} I-A\right)^{-1} x^{(l)}$ and $\hat{\beta}^{(l)}=\left\|\left(\alpha^{(l)} I-A\right)^{-1} x^{(l)}\right\|_{2}$.

We describe two algorithms for simultaneously finding the eigenpairs of an $n \times n$ real symmetric matrix $A$. This method is a good choice if $\|S-A\|_{F}<$ $\operatorname{gap}^{*}(S)$, where $S$ is the initial matrix.

\subsection{Parameterized Newton's Method with Orthogonalization}

Let $A=\left(a_{i j}\right) \in M_{n}$ be a given symmetric matrix with eigenpairs $\left(\begin{array}{l}u_{k} \\ \lambda_{k}\end{array}\right)$ for $k=$ $1, \ldots, n$.The following algorithm simultaneously computes $\left(\begin{array}{c}u_{k} \\ \lambda_{k}\end{array}\right), k=1, \ldots, n$, using the parameterized Newton's Method with orthogonalization. 
Choose $S=\operatorname{diag}\left(\alpha_{1}^{(0)}, \ldots, \alpha_{n}^{(0)}\right)$ be the initial matrix where the diagonal elements of $S$ are arranged in decreasing order. Let $\epsilon>0$ be the given tolerance. Step 1: Set the initial eigenpairs as $\mathcal{D}=\left\{\left(\begin{array}{c}x_{k}^{(0)} \\ \alpha_{k}^{(0)}\end{array}\right)\right\}$ for $k=1, \ldots, n$ where $x_{k}^{(0)}$ is the $k$ th column of identity matrix.

Step 2: For $k=1, \cdots, n$ do (in parallel) (i) Compute $\left\|\left(\alpha_{k}^{(0)} I-A\right) x_{k}^{(0)}\right\|_{2}$ (ii) If $\left\|\left(\alpha_{k}^{(0)} I-A\right) x_{k}^{(0)}\right\|_{2}<\epsilon$, then go to Step 5 , otherwise go to Step 3 .

\section{End}

\section{Step 3: Parameterized Newton's Iteration:}

For $k=1, \cdots, n$ do (in parallel),

$3($ a) For $i=0,1, \cdots$ do until convergence

(i) Solve $\left(\alpha_{k}^{(i)} I-A\right) y_{k}^{(i)}=x_{k}^{(i)}$. (ii) Compute $\beta_{k}^{(i)}=\left(x_{k}^{(i)}\right)^{T} y_{k}^{(i)}$.

(iii) Compute $\hat{\beta}_{k}^{(i)}=\left\|y_{k}^{(i)}\right\|_{2}$. (iv) $x_{k}^{(i+1)}=\frac{1}{\hat{\beta}^{(i)}} y_{k}^{(i)}$.

(v) Compute $p_{i}=\frac{\beta_{k}^{(i)}}{\hat{\beta}_{k}^{(i)}}$. (vi) If $\left(1-\left|p_{i}\right|\right)<\epsilon$ go to $3(\mathrm{~b})$.

If $\left|p_{i}\right|<\epsilon$ go to $3(\mathrm{c})$ (vii) Compute $\alpha_{k}^{(i+1)}=\alpha_{k}^{(i)}-\frac{1}{\hat{\beta}_{k}^{(i)}} p_{i}$.

$3(\mathrm{~b})$ Set $\lambda_{k}=\alpha_{k}^{(i)}-\frac{1}{\hat{\beta}_{k}^{(i)}}, u_{k}=x_{k}^{(i+1)} 3(\mathrm{c})$ Set $\lambda_{k}=\alpha_{k}^{(i)}-\frac{1}{\hat{\beta}_{k}^{(i)}}$

and then compute the corresponding normalized eigenvector $u_{k}$ of $A$. End

End.

Step 4: Let $m$ be the number of eigenpairs obtained from Step 3. If $m=n$, then all $n$ distinct eigenpairs have been obtained, go to Step 5 . If $m<n$, then obtain $(n-m)$ remaining eigenpairs as follows: 4 -(i) Denote by $\left\{\hat{G}_{k}\right\}_{k=1, \ldots, m}$ the $m$ groups of eigenpairs as defined below.

$$
\hat{G}_{k}=\left\{\left(\begin{array}{c}
x_{k j}^{(0)} \\
\alpha_{k j}^{(0)}
\end{array}\right) /\left(\begin{array}{c}
x_{k j}^{(0)} \\
\alpha_{k j}^{(0)}
\end{array}\right) \text { converges to }\left(\begin{array}{c}
u_{k} \\
\lambda_{k}
\end{array}\right)\right\} \text { for } j=1, \ldots s_{k}
$$

where $s_{1}+s_{2}+\cdots+s_{m}=n$ and $s_{k}$ is the number of eigenpairs in a Group $\hat{G}_{k}$. Set $p=$ number of Groups of $\hat{G}_{k}$ having number of eigenpairs $s_{k}>1$. If $p=0$ then go to Step 5.

4-(ii) For each of the above $p$ groups do the following steps, in parallel.

Compute: $\min _{1 \leq j \leq s_{k}}\left\|\left(\alpha_{k_{j}}^{(0)} I-A\right) X_{k_{j}}^{(0)}\right\|_{2}$. Discard one eigenpair which achieves the minimum (Note that there is one eigenpair in each group that achieves the minimum).

4 -(iii) Orthogonalize the other vectors in each of the above $p$ groups (in parallel): Set $l=m$. For $j=1, \ldots,\left(s_{k}-1\right)$ do

Orthogonalize $\left\{u_{1}, u_{2}, \ldots, u_{l}, x_{k_{j}}^{(0)}\right\} \longrightarrow\left\{u_{1}, u_{2}, \ldots, u_{l}, \hat{x}_{k_{j}}^{(0)}\right\}$ using the modified Gram-Schmidt process. 
(a) Using Step 3 with eigenpair $\left(\begin{array}{c}\hat{x}_{k_{j}}^{(0)} \\ \alpha_{k_{j}}^{(0)}\end{array}\right)$, obtain a new eigenpair $\left(\begin{array}{c}\hat{x}_{k_{j}} \\ \hat{\alpha}_{k_{j}}\end{array}\right)$.

(b) Set $\left(\begin{array}{c}\hat{x}_{k_{j}} \\ \alpha_{k_{j}}\end{array}\right) \rightarrow\left(\begin{array}{c}u_{l+1} \\ \lambda_{l+1}\end{array}\right)$. (c) $l \leftarrow l+1$. End

4-(iv) Let $p^{\prime}=$ number of distinct eigenpairs obtained in 4(iii) where $p^{\prime} \leq$ $(n-m)$.Set $m \leftarrow m+p^{\prime}$ If $m<n$ go to 4(i) otherwise go to step 5. Step 5. End.

Table 1. $(q=4, n=50)$

\begin{tabular}{|c|c|c|c|c|c||c|}
\hline eigenvalues & $t_{0}$ & $t_{1}$ & $t_{2}$ & $t_{3}$ & $t_{4}$ & Eigenvalues \\
\cline { 2 - 6 } of $A\left(t_{i}\right)$ & 0 & 0.25 & 0.5 & 0.75 & 1.0 & from MATLAB \\
\hline 1 st & 150.00 & 457.8705 & 879.5562 & 1308.3704 & 1739.0537 & 1739.0537 \\
$\vdots$ & $\vdots$ & $\vdots$ & $\vdots$ & $\vdots$ & $\vdots$ & $\vdots$ \\
20 th & 36.00 & 32.0262 & 32.6110 & 27.9100 & 23.4161 & 23.4161 \\
$\vdots$ & $\vdots$ & $\vdots$ & $\vdots$ & $\vdots$ & $\vdots$ & $\vdots$ \\
40 th & -87.00 & 94.4645 & -96.4081 & -104.6880 & -112.8265 & -112.8265 \\
$\vdots$ & $\vdots$ & $\vdots$ & $\vdots$ & $\vdots$ & $\vdots$ & $\vdots$ \\
50 th & -147.00 & -164.1900 & -215.4465 & -285.9817 & -363.2445 & -363.2445 \\
\hline M & & 46 & 44 & 45 & 45 & \\
\hline B & & 4 & 6 & 5 & 5 & \\
\hline C(D) & & $8(6)$ & $8(5)$ & $10(4)$ & $8(4)$ & \\
\hline
\end{tabular}

\subsection{The Group Homotopy Algorithm with Parameterized Newton's Iteration}

Given an $n \times n$ symmetric matrix $A$, the following algorithm simultaneously computes the eigenpairs $\left(\begin{array}{c}u_{k} \\ \lambda_{k}\end{array}\right)$, for $k=1, \ldots, n$ of $A$, using the Group Homotopy Method.

Step 0: $\quad$ Set $t_{0}=0$. Set $S=A(0)=\operatorname{diag}\left(\alpha_{1}^{(0)}, \ldots, \alpha_{n}^{(0)}\right)$, where $\alpha_{k}^{(0)}, k=$ $1, \ldots, n$ are arbitrarily chosen real numbers and the corresponding initial eigenvectors are the columns of the identity matrix.

Step 1: Choose $q \geq 4$. Set $\Delta t=\frac{1}{q}$.

Step 2: For $i=1, \ldots, q$ do (i)Compute $t_{i}=t_{i-1}+\Delta t$ (ii) If $t_{i} \geq 1$ then set $t_{i}=1$ (iii) Apply Algorithm 3.1 to obtain all the eigenpairs of $A\left(t_{i}\right)=$ $A\left(t_{i-1}\right)+\Delta t(A-A(0))$ (iv)If $t_{i}<1$ go to step 2(i) otherwise stop. End.

Numerical Results. Since $\|\cdot\|_{2} \leq\|\cdot\|_{F}$ where $\|\cdot\|_{F}$ is the Frobenius matrix norm and $\|\cdot\|_{F}$ is easy to compute, we use $\|A-S\|_{F}$ to compute $\Delta t_{i}$ in the following examples. Let $\mathrm{M}=$ the number of eigenpairs obtained using the modified Newton iteration. $\mathrm{B}=$ the number of eigenpairs recovered with modified Gram-Schmidt process using the modified Newton iteration. $\mathrm{C}=$ the maximum 
number of iterations at each step. $\mathrm{D}=$ the maximum number of iterations needed for the orthogonalization.

Example 1: Consider the matrix

$A=\left[a_{i j}\right], \in M_{n}, a_{i j}=i$ if $i>j, a_{i j}=(-1)^{i} 3 * i$ if $\mathrm{i}=\mathrm{j}, a_{i j}=j$ if $j>i$.

Choose the initial matrix $S$ such that $A(0)=S=\operatorname{diag}\left(a_{11}, a_{22}, \ldots, a_{n n}\right)$.

$\|A-A(0)\|_{F}=1779.2$. The result of Table 1 uses $q=4$. From the results of numerical experiments below, it is clear that if $q$ is large then the number of iterations in each step is significantly lower (see last row of each table) and also the number of eigenpairs recovered using algorithm 3.1 (Step 4) is also less (row representing $B$ ).

Since $\operatorname{Gap}^{*}<\operatorname{gap}^{*}(S)$, we choose $\Delta t=1$. It shows that the choice of $S$ is very important to accomplish the experiment in an efficient way.

\section{Conclusions}

We have presented a new Homotopy method, called the Group Homotopy method for computing the eigenvalues and eigenvectors of a real symmetric matrix. The important features of this method are:

(i) Unlike the other methods for symmetric eigenvalue problem, the new method does not transform the initial matrix to a tridiagonal matrix, and is thus suitable for large and sparse problems.

(ii) The method can take advantage of the structure preserving linear systems solvers, especially designed for structure matrices, such as Toeplitz. Thus the method is expected to be much faster than the existing methods in most cases.

(iii) Unlike the other existing Homotopy methods, the step-size is predetermined at the outset according to a criterion presented in the paper and is never altered at any iteration during the execution of the algorithm.

(iv) The algorithm is parallel in nature.

\section{References}

1. M.T. Chu : On a Numerical Treatment for the Curve-Tracing Of the Homotopy Method. Numerische Mathematik, 42.(1983) 323-329

2. M.T. Chu : A Simple Application of the Homotopy Method to Symmetric Eigenvalue Problems. Linear Algebra Appl., 59.(1985) 85-90

3. M.T. Chu : A Note on The Homotopy Method for Linear Algebraic Eigenvalue Problems. Linear Algebra Appl., 108.(1988) 225-236

4. Karabi Datta and Yoopyo Hong and Ran Baik Lee : An Application of Homotopy Method For Eigenvalue Problem of A Symmetric Matrix. IMACS, Series in Computational and Applied Mathematics, 3.(1996) 367-376

5. Karabi Datta and Yoopyo Hong and Ran Baik Lee : Parameterized Newton's iteration for computing an Ejgenpairs of a real Symmetric Matrix in an Interval, Computational Methods in Applied Mathemaics, 3.(2003) 517-535 
6. Roger A. Horn and Charles R. Johnson: Matrix Analysis. Cambridge University Press, (1985)

7. K. Li and T.Y. Li: An Algorithm for Symmetric Tridiagonal Eigen Problems: Divide and Conquer with Homotopy Continuation. SIAM J. Sci. Comput., 14.(1993) 735751

8. Beresford N. Parlett: The Symmetric Eigenvalue Problem. Prentice-Hall, Inc.Englewood Cliffs, (1980) 